\title{
RELATIONS ENTRE LES NOTIONS DE LIMITE ET DE DISTANCE*
}

\author{
PAR

\section{MAURICE FRÉCHET}

\section{Position DU PROBLÈme}

1. Un certain nombre de propriétés des ensembles linéaires peuvent être étendues aux ensémbles abstraits, c'est à dire aux ensembles dont les éléments sont de nature quelconque ou inconnue.

C'est ainsi que G. Cantor a pu développer la théorie des nombres cardinaux et ordinaux qui s'applique aussi bien aux ensembles abstraits qu'aux ensembles linéaires. C'est ainsi qu'on peut donner une définition de l'intégrale d'une fonction étendue à un ensemble abstrait en généralisant d'une façon presque immédiate une définition donnée par J. Radon pour l'intégrale étendue à un espace à un nombre fini de dimensions $\dagger$ comme je l'ai montré récemment.ł

2. Mais si l'on veut aller plus loin, on est amené à introduire la notion de limite ou même la notion de distance tout en s'efforçant de les présenter sous une forme qui ne fasse pas intervenir la nature des éléments de la classe d'éléments considérée.

En ce qui concerne la notion de limite, on peut se contenter d'admettre qu'il existe une règle d'ailleurs quelconque ou inconnue permettant de décider si une suite infinie d'éléments $A_{1}, A_{2}, \cdots, A_{n}, \cdots$, de la classe considérée converge ou non vers un élément déterminé $A$. On se bornera à supposer que ce critère est tel que $1^{\circ}$ si les éléments de la suite sont identiques, la suite converge et converge vers $A_{1} ; 2^{\circ}$ si une suite $\left\{A_{n}\right\}$ converge vers $A$, toute suite infinie extraite de la suite $\left\{A_{n}\right\}$ converge aussi vers $A$.

De telles classes d'enscmbles, que j'ai appelées classes $(L)$, ne sont pas moins générales qu'une classe abstraite quelconque. Rien n'empêche en effet de considérer une classe abstraite arbitraire comme classe $(L)$ en s'y prenant par exemple de la manière suivante: on porte son attention sur une suite arbitraire $A, A_{1}, A_{2}, \cdots, A_{n}, \cdots$. Une suite d'éléments distincts sera considérée comme convergente si c'est la suite $A_{1}, A_{2}, \cdots, A_{n}, \cdots$, ou si elle est extraite de cette suite, et dans les deux cas la suite est dite converger

\footnotetext{
* Presented to the Society, April, 1917.

$\dagger$ J. Radon, W i e n e r S i t z ung s be r i c h t e, vol. 122, $2 a$ (1913), pp. 1295-1438.

$\ddagger$ M. Fréchet, Bulletin de la Société Mathématique de France, vol. 43 (1915), pp. 248-265.
} 
vers l'élément $A$. Dans le cas contraire, la suite est considérée comme divergente.

Ceci étant et moyennant une extension convenable des définitions ordinaires, on peut développer une théorie de la connexion des ensembles abstraits dans les classes où une telle définition de la limite est adoptée* ou encore généraliser quelques théorèmes sur les fonctions également continues. $\dagger$

3. Mais si l'on veut généraliser des théorèmes plus précis de la théorie des fonctions, une définition aussi indéterminée de la limite ne suffit plus. J'ai donc été amené, dans ma Thèse, à étudier l'effet de restrictions de plus en plus grandes apportées à l'idée de limite, ces restrictions restant toutefois telles que toutes les définitions classiques de la limite dans les ensembles les plus importants (ensembles de points, de courbes, de fonctions continues, etc.) y satisfont par avance.

La notion qui intervient de la façon la plus essentielle pour faciliter l'étude de la limite dans la théorie des ensembles linéaires est la notion de distance. Réduite à ses caractéristiques abstraites, on peut la décrire ainsi:

Dans une classe $(L)$, on peut définir la distance si à tout couple $A, B$ d'éléments de la classe correspond un nombre $(A, B)=(B, A) \geqq 0$ jouissant des propriétés suivantes:

$1^{\circ}(A, B)$ n'est nul que si $A, B$ coïncident;

$2^{\circ}$ si, $A$ restant fixe, la suite $\left\{B_{n}\right\}$ converge vers $A,\left(A, B_{n}\right)$ doit converger vers zéro et réciproquement;

$3^{\circ}$ on a, quels que soient les éléments $A, B, C$ de la classe,

$$
(B, C) \leqq(A, B)+(A, C) \text {. }
$$

J'appellerai un tel nombre la distance de $A$ à $B$ et je dirai d'une classe $(L)$ où on peut définir une distance que c'est une classe $(D)$. $\ddagger$

Il faut tout d'abord remarquer que les conditions $1^{\circ}, 2^{\circ}, 3^{\circ}$ à elles seules ne peuvent apporter aucune limitation à l'arbitraire de la nature des éléments de la classe. Considérons en effet la catégorie la plus générale des classes d'éléments abstraits. On peut d'abord définir au moins d'une facon une nombre satisfaisant à $1^{\circ}, 3^{\circ}$-il suffit par exemple de prendre pour $(A, B)$ la valeur 1 quand $A, B$ sont distincts, 0 quand ils coïncident. $\S$ Une fois choisie une définition de la distance satisfaisant à $1^{\circ}$ et $3^{\circ}$, si l'on définit précisément la limite par la condition $2^{\circ}$, on aura bien une classe $(D)$.

4. Le problème qui se pose n'est donc pas de savoir à quelle condition une classe abstraite est une classe $(D)$, mais à quelles conditions simples et

* M. Fréchet, M a the matis c he A n n al en, vol. 68 (1910), pp. 145-168.

$\dagger \mathrm{M}$. Fréchet, Thèse, Rendiconti del Circolo Matematico di Pale r m o, vol. 22 (1906), pp. 1-74.

$\ddagger$ Je modifie donc ici et dans la suite les dénominations adoptées dans ma Thèse. Ce que j'y ai appelé écart et voisinage je l'appellerai ici distance et écart uniformément régulier.

$\S$ Voir Note Supplémentaire $A$ à la fin de cette memoire. 
indépendantes une classe $(L)$ où les suites convergentes et leurs limites sont déjà définies est-elle une classe $(D)$ et comment on y peut calculer la distance $(A, B)$.

Ainsi, l'objet de ce travail est le suivant: sachant qu'on peut toujours de bien des façons définir dans une classe d'éléments abstraits les suites convergentes et leurs limites, il s'agit de déterminer à quelles conditions supplémentaires il faut assujettir ce choix pour que l'on puisse défnir sur cette classe une distance telle que la convergence défnie d'avance ne soit pas modifiée quand on la défnit au moyen de cette distance.

\section{RÉDUCTION DU PROBLÈME}

5. Pour résoudre ce problème, nous porterons d'abord notre attention sur les conditions $1^{\circ}$ et $2^{\circ}$. Si dans une classe $(L)$, on parvient à définir un nombre $(A, B)=(B, A) \geqq 0$ satisfaisant aux conditions $1^{\circ}$ et $2^{\circ}$, on dira que cette classe est une classe $(E)$, ou encore qu'on a pu y définir un écart, ${ }^{*}$ à savoir le nombre $(A, B)$, qui sera l'écart des éléments $A$ et $B$. Si cet écart satisfait en même temps à la condition $3^{\circ}$, ce sera en même temps une " distance."

D'autre part il est facile de vérifier que dans une classe $(D)$ tout ensemble dérivé est fermé, c'est à dire que si une suite d'éléments $\left\{A_{n}\right\}$ converge vers l'élément $A$ et si chaque élément $A_{n}$ est la limite d'une suite convergente $A_{1}^{(n)}, A_{2}^{(n)}, \cdots, A_{p}^{(n)}, \cdots$, on peut extraire de l'ensemble à double entrée $\operatorname{des} A_{p}^{(n)}$ une suite $A_{p_{1}}^{\left(n_{1}\right)}, A_{p_{2}}^{\left(n_{2}\right)}, \cdots$, qui converge vers $A$. Car

$$
\left(A, A_{p_{i}}^{\left(n_{i}\right)}\right) \leqq\left(A, A_{n_{i}}\right)+\left(A_{n_{i}}, A_{p_{i}}^{\left(n_{i}\right)}\right),
$$

et en prenant $n_{i}$ puis $p_{i}$ assez grand, on rend le second membre et par suite le premier aussi petit que l'on veut.

Nous dirons d'une classe $(L)$ où tout ensemble dérivé est fermé que c'est une classe $(S)$.

6. Nous avons dès lors obtenu deux conditions nécessaires pour qu'une classe $(L)$ admette une définition de la distance, c'est à dire soit une classe $(D)$. Il faut que cette classe $(L)$ soit une classe $(E)$ et en même temps une classe $(S)$.

Il est facile de voir que ces deux conditions sont indépendantes.

Considérons en effet la classe formée des points d'un plan définis comme il suit par leurs coordonnées cartésiennes:

pour le point $A_{0}: x=0, y=0$;

pour un quelconque $A_{n}$ des points $A_{1}, A_{2}, \cdots, A_{n}, \cdots: x=1 / n, y=0$; pour un quelconque $A_{p}^{(n)}$ des points de la suite $A_{1}^{(1)}, A_{1}^{(2)}, A_{2}^{(1)}, \cdots$, ̀̀ double entrée: $x=1 / n, y=1 / p$.

* J'introduis ici une notation $(E)$ et un mot "écart" que j'ai employé dans ma Thèse, là où j'emploie maintenant la notation $(D)$ et le mot distance. 
Nous pouvons définir ainsi un écart de deux points quelconques de la classe; il sera pris égal:

à l'unité si l'un des points est $A_{0}$ et l'autre l'un des points $A_{p}^{(n)}$;

à la distance géométrique des deux points sur le plan dans tout autre cas. Ceci étant nous pouvons considérer la classe comme une classe $(L)$ et en même temps comme une classe $(E)$ en y définissant la limite précisément par la condition $2^{\circ}$ énoncée plus haut.

Cependant elle ne peut être une classe $S$, bien évidemment.

Inversement une classe $(S)$ peut ne pas être une classe $(E)$. En effet reprenons la même classe d'éléments, mais cette fois définissons autrement la convergence d'une suite. Une suite d'éléments de la classe sera dite convergente vers l'un d'eux lorsque leur distance géométrique à ce dernier tend vers zéro, à moins que la suite ne comprenne les deux points $A_{1}^{(1)}, A_{1}^{(2)}$, cas où la suite sera dite divergente. Il est évident que dans cette classe tout ensemble dérivé y est fermé. Pourtant, on ne peut y définir un écart sans altérer la convergence des suites. Car dans ce cas, si on considère la suite $A_{1}, A_{2}, \cdots, A_{n}, \cdots$, par exemple, elle converge vers $A_{0}$, donc si l'écart existait, la suite $\left(A_{0}, A_{1}\right),\left(A_{0}, A_{2}\right), \cdots,\left(A_{0}, A_{n}\right), \cdots$, devrait converger vers zéro. Par suite, il en serait de même de la suite de nombres $\left(A_{0}, A_{1}^{(1)}\right)$, $\left(A_{0}, A_{1}^{(2)}\right),\left(A_{0}, A_{1}\right),\left(A_{0}, A_{2}\right), \cdots,\left(A_{0}, A_{n}\right), \cdots$, et alors la suite de points $A_{1}^{(1)}, A_{1}^{(2)}, A_{1}, A_{2}, \cdots, A_{n}, \cdots$, convergerait vers $A_{0}$ contrairement à l'hypothèse.

7. Nous remarquons immédiatement que les exemples précédents montrent en même temps que si l'on a défini d'avance la limite dans une classe abstraite, il n'est pas toujours possible d'y définir la distance de deux éléments quelconques de façon que la limite antérieurement définie puisse aussi être définie sans altération au moyen de la distance. Nous voyons aussi qu'il n'est même pas toujours possible d'y définir un écart sans altérer les limites.

8. Nous pouvons en outre décomposer en plusieurs autres conditions indépendantes la condition pour une classe $(L)$ d'être une classe $(E)$.

Si une classe est $(E)$, la condition nécessaire et suffisante pour qu'une suite d'éléments $\left\{A_{n}\right\}$ converge vers l'élément $A$ est que pour $q$ donné on puisse trouver $p$ tel que

$$
\left(A_{n}, A\right)<\frac{1}{q}
$$

pour $n>p$. Autrement dit si l'on appelle $\Sigma_{q}$ l'ensemble des éléments $B$ tels que $(A, B)<1 / q$, il faut et il suffit que quel que soit $q$, la suite $\operatorname{des} A_{n}$ soit contenue dans $\Sigma_{q}$ à partir d'un certain rang variable avec $q$.

Remarquons d'ailleurs que $\Sigma_{q+1}$ fait partie de $\Sigma_{q}$ et que $\Sigma_{1}, \Sigma_{2}, \cdots$ ont un seul élément commun savoir $A$.

On est alors amené à la définition suivante. Soit une classe $(L)$; on dira 
que c'est une classe $(V)$ ou encore qu'on peut y définir un voisinage* lorsque la condition suivante est réalisée:

Pour tout élément $A$, il existe une suite d'ensembles $U_{1}, U_{2}, \cdots, U_{q}, \cdots$ telle que la condition nécessaire et suffisante pour qu'une suite. $\left\{A_{n}\right\}$ converge vers $A$ est que quel que soit $q$, la suite $\left\{A_{n}\right\}$ fasse partie de l'ensemble $U_{q}$ à partir d'un certain rang (variable en général avec $q$ ).

On remarque aussitôt, que s'il en est ainsi l'élément $A$ est le seul élément commun à $U_{1}, U_{2}, \cdots, U_{q}, \cdots$, et qu'en appelant $T_{q}$ l'ensemble $U_{1} \cdot U_{2}$. $\cdots \cdot U_{q}$ des éléments communs à $U_{1}, U_{2}, \cdots, U_{q}$, l'ensemble $T_{q}$ est contenu dans $T_{q-1}$ et la suite des ensembles $T_{1}, T_{2}, \cdots, T_{q}, \cdots$, peut remplacer la suite des ensembles $U_{1}, U_{2}, \cdots, U_{q}, \cdots$.

Ainsi on peut supposer que $U_{q}$ appartienne à $U_{q-1}$ et on doit se souvenir que $A$ est le seul élément commun aux $U_{q}$. Lorsqu'il y a lieu de distinguer les $U_{q}$ relatifs à un élément $A$ de ceux relatifs à un élément $B$, on désignera les premiers par $U_{q}^{(A)}$ et les seconds par $U_{q}^{(B)}$.

Nous pourrons donc dire qu'un élément $B$ est très voisin de $A$ lorsque $B$ est dans un ensemble $U_{q}^{(\Lambda)}$ d'indice très grand. Ou encore nous pouvons dire que les ensembles $U_{1}^{(A)}, U_{2}^{(A)}, \cdots$, constituent successivement des voisinages de plus en plus étroits de $A$.

9. Nous avons alors à résoudre la question suivante: soit une classe $(L)$ où les suites convergentes et leurs limites sont déterminées d'avance, est-il toujours possible d'assigner à chaque élément $A$ une suite d'ensembles $T_{1}^{(\Lambda)}, T_{2}^{(\Lambda)}, \cdots$, déterminant des voisinages de plus en plus étroits de $A$, de sorte que les suites convergentes et leurs limites ne soient pas modifiées?

Nous avons aussi à résoudre la question suivante: si dans une classe $(V)$ le voisinage autour de chaque élément est défini d'une certaine façon, est-il toujours possible d'assigner un écart à tout couple d'éléments de l'ensemble, de sorte que la convergence et la limite éventuelle d'une suite ne soient pas modifiées, qu'on définisse celles-ci par le moyen du voisinage ou par celui de l'écart?

Nous allons voir que la réponse à ces deux questions est négative et nous allons déterminer quelles sont les conditions supplémentaires que doit vérifier la définition de la limite pour qu'on puisse la définir au moyen soit du voisinage, soit de l'écart.

10. La réponse à la première question est négative, c'est à dire qu'une classe $(L)$ étant donnée, on ne peut pas toujours y définir un voisinage* sans altérer la convergence ou la limite de ses suites d'éléments.

Pour qu'une classe $(L)$ puisse être considérée comme une classe $(V)$, sans modifier la convergence des suites d'éléments ni les limites des suites convergentes, il faut et il suffit que:

* J'emploie ici la notation $(V$ ) et le mot de voisinage dans un sens plus général que celui que avais adopté dans ma Thèse. Voir aussi Note Supplémentaire $B$ à la fin de cette mémoire. 
$1^{\circ}$ toute suite convergente d'éléments de la classe $(L)$ reste convergente quand on lui ajoute un nombre fini d'éléments de la classe;

$2^{\circ}$ pour tout élément non isolé $A$ de la classe, toute suite $\sigma$ d'éléments $A_{1}^{(\sigma)}, A_{2}^{(\sigma)}, \cdots$, convergeant vers $A$ et tout entier $n$, il existe un entier $q_{n}^{(\sigma)}$ jouissant de la propriété suivante: Soient $\sigma_{1}, \sigma_{2}, \cdots$, une suite de suites (distinctes ou non) convergeant vers $A$ et $n_{1}, n_{2}, \cdots$, une suite d'entiers. Si quel que soit l'entier $N$ on peut trouver l'entier $i_{0}$ tel que pour $i>i_{0}$, $n_{i}>q_{N}^{\left(\sigma_{i}\right)}$, alors la suite $A_{n_{1}}^{\left(\sigma_{1}\right)}, A_{n_{2}}^{\left(\sigma_{2}\right)}, A_{n_{s}}^{\left(\sigma_{3}\right)}, \cdots$, converge aussi vers $A$.

Ces conditions sont nécessaires. Car si la classe est $(V)$, la limite $A$ d'une suite $A_{1}, A_{2}, \cdots$, est définie de la façon suivante. Il existe des ensembles $T_{1}, T_{2}, \cdots, T_{r}, \cdots$, chacun dans le précédent, n'ayant en commun que l'élément $A$, tels que si $A_{1}, A_{2}, \cdots$, converge vers $A$, cette suite est contenue dans chaque $T$, à partir d'un certain rang. S’il en est ainsi, cette condition sera encore réalisée si on ajoute un nombre fini d'éléments de la classe à la suite, donc la convergence et la limite de cette suite n'en seront pas modifiées. D'autre part soit $q_{n}^{(\sigma)}$ le rang du premier élément de la suite $A_{1}^{(\sigma)}, A_{2}^{(\sigma)}, \cdots$, $A_{n}^{(\sigma)}, \cdots$, à partir duquel les termes de cette suite supposée convergeant vers $A$, sont tous compris dans $T_{n}$. Et supposons que quel que soit $N$ on peut trouver $i_{0}$ tel que $n_{i}>q_{N}^{\left(\sigma_{i}\right)}$ pour $i>i_{0}$. Alors $A_{n_{i}}^{\left(\sigma_{i}\right)}$ est dans $T_{N}$ pour $i>i_{0}$; autrement dit, quel que soit $N$, la suite $A_{n_{1}}^{\left(\sigma_{1}\right)}, A_{n_{2}}^{\left(\sigma_{2}\right)}, \cdots$, appartient à $T_{N}$ à partir d'un certain rang $i_{0}$ variable avec $N$. Elle est donc convergente et sa limite est $A$.

Inversement, les conditions $1^{\circ}$ et $2^{\circ}$ sont suffisantes pour que la classe $(L)$ soit une classe $(V)$.

En effet; tout élément $B$ de la classe appartient à au moins une suite convergeant vers $A$ (par exemple $B, A, A, A, \cdots, A, \cdots)$; soit $A_{1}^{(\sigma)}, A_{2}^{(\sigma)}$, $\cdots, A_{p-1}^{(\sigma)}, B, A_{p+1}^{(\sigma)}, \cdots$, cette suite. Nous appellerons $U_{n}^{(\boldsymbol{A})}$ l'ensemble des éléments $B$ tels que dans une au moins des suites $\sigma$ tendant vers $A$ et comprenant $B$ celui-ci occupe un rang supérieur à $q_{n}^{(\sigma)}$. Les ensembles $U_{n}^{(\boldsymbol{A})}$ jouissent alors des propriétés voulues. Considérons d'abord une suite $A_{1}^{(\sigma)}$, $A_{2}^{(\sigma)}, \cdots, A_{p}^{(\sigma)}, \cdots$, convergeant vers $A$. Quel que soit $n$, l'élément $A_{p}^{(\sigma)}$ occupera pour $p$ assez grand $\left(p \geqq p_{0}\right)$ un rang supérieur à $q_{n}^{(\sigma)}$. Donc les éléments de cette suite à partir de $A_{p_{0}}$ appartiennent à $U_{n}^{(A)}$. Inversement si les éléments de cette suite appartiennent quel que soit $N$ à $U_{N}^{(\Lambda)}$ à partir d'un certain rang $p_{0}$ (variable avec $N$ ) c'est que pour $p \geqq p_{0} . \quad A_{p}^{(\sigma)}$ appartient à au moins une suite $\left(\sigma_{p}\right)$ convergeant vers $A$ où il occupe un rang $r_{p}>q_{N}^{\left(\sigma_{p}\right)}$. Autrement dit, étant donnée la suite

$$
A_{1}^{(\sigma)}=A_{r_{1}}^{\left(\sigma_{1}\right)}, \quad A_{2}^{(\sigma)}=A_{r_{2}}^{\left(\sigma_{2}\right)}, \quad \cdots, \quad A_{p}^{(\sigma)}=A_{r_{p}}^{\left(\sigma_{p}\right)}, \quad \cdots,
$$

on peut pour chaque valeur de $N$ trouver $p_{0}$, tel que $p \geqq p_{0}$ entraine $r_{p}>q_{N}^{\left(\sigma_{p}\right)}$ et alors d'après la condition $2^{\circ}$ la suite précédente converge vers $A$. 
11. Les conditions $1^{\circ}$ et $2^{\circ}$ sont d'ailleurs indépendantes.

On peut satisfaire à $2^{\circ}$ sans satisfaire à $1^{\circ}$ en prenant par exemple comme classe les points d'un segment $B C$ extrémités comprises et en y définissant la limite comme d'ordinaire par la distance sauf que les suites comprenant le point $B$ et dont la distance des points à $B$ ne tend pas vers zéro seront toujours déclarées divergentes dans cette classe. Alors si on considère une suite convergeant vers $A$ et si on lui adjoint $B$, elle cesse d'être convergente; $1^{\circ}$ cesse d'être vérifié. $\mathrm{Au}$ contraire $2^{\circ}$ est encore vérifié. Soit en effet une suite $A_{1}^{(\sigma)}, A_{2}^{(\sigma)}, \cdots$, convergeant vers $A$. On appellera $q_{n}^{(\sigma)}$ le rang a partir duquel on a constamment $A A_{p}^{(\sigma)}<1 / n$. Alors si quel que soit $N$, on peut trouver $i_{0}$, tel que pour $i>i_{0}$, on ait $n_{i}>q_{N}^{\left(\sigma_{i}\right)}$, c'est que $A A_{n_{i}}^{\left(\sigma_{i}\right)}<1 / N$ pour $i>i_{0}$. Si $A \neq B$, aucune des suites $\sigma_{i}$, supposées convergeant vers $A$, ne contiendra $B$; par suite la suite $A_{n_{1}}^{\left(\sigma_{1}\right)}, A_{n_{2}}^{\left(\sigma_{2}\right)}, \cdots$, non plus, et alors elle convergera vers $A$ dans la classe. Si $A=B$, la présence de $B$ dans les suites n'offre plus de difficulté.

On peut aussi satisfaire à $1^{\circ}$ sans satisfaire à $2^{\circ}$.

Nous allons donner un exemple d'une classe $(L)$ où la limite est définie de façon a satisfaire aux trois conditions $1^{\circ}, 2^{\circ}$ du $n^{\circ} 3$ et $1^{\circ}$ du $n^{\circ} 10$ sans qu'on puisse y définir un voisinage non seulement au sens de ma Thèse mais même au sens plus large adopté ici $\left(n^{\circ} 8\right)$. Il suffit de montrer que la condition $2^{\circ}$ $\mathrm{du} \mathrm{n}^{\circ} 10$ n'est pas satisfaite.

Cet exemple est celui d'une classe $(L)$ dont les éléments sont les fonctions $f(x)$ uniformes sur le segment $J(0 \leqq x \leqq 1)$ et où la limite est définie comme d'ordinaire. C'est à dire que $f_{n}(x)$ converge vers $f(x)$ si ceci a lieu en chaque point de l'intervalle (uniformément ou non). Alors les trois conditions mentionnées plus haut sont satisfaites. Il faut montrer que la dernière condition $2^{\circ}$ ne l'est pas.*

Prenons pour simplifier le cas où l'élément $A$ est la fonction zéro (c'est à dire égale à zéro en tout point de l'intervalle $J$ ). Montrons que si pour chaque entier $N$ et pour chaque suite $o$ de fonctions convergeant vers zéro, $f_{1}^{\sigma}(x), f_{2}^{\sigma}(x), \cdots$, on détermine un certain entier $q_{N}^{\sigma}$, aucun choix de l'ensemble des entiers $q_{N}^{\sigma}$ ne satisfera à la condition $2^{\circ}$. Considérons en particulier la suite $\sigma_{n}$ formée des fonctions $f_{1}^{n}(x), f_{2}^{n}(x), \cdots, f_{p}^{n}(x), \cdots$, de la manière suivante. La fonction $y=f_{p}^{n}(x)$ est de période $1 / 2^{n}$ et elle est représentée par une ligne brisée dont les premiers sommets sont les points de coordonnées

$$
(0,0) ; \quad\left(\frac{1}{2^{n+p+1}}, 2\right) ; \quad\left(\frac{1}{2^{n+p}}, 0\right) ; \quad\left(\frac{1}{2^{n}}, 0\right) .
$$

On voit que les éléments de la suite $\sigma_{n}$ convergent vers zéro quand $p$ croît indéfiniment. $\overline{\mathrm{A}} \sigma_{n}$ et $N$ correspond un certain entier $q_{N}^{\sigma_{n}}$; posons

* Voir Note Supplémentaire $C$ à la fin de cette mémoire. 


$$
p_{n}=1+q_{1}^{\sigma_{n}}+q_{2}^{\sigma_{n}}+\cdots+q_{n}^{\sigma_{n}} .
$$

Extrayons des suites $\sigma_{n}$ la suite $\sigma$ :

$$
f_{p_{1}}^{1}, f_{p_{2}}^{2}, \cdots, f_{p_{n}}^{n}, \cdots \text {. }
$$

Pour chaque valeur de $N$ on peut trouver $i$ tel que pour $n>i$ on ait $p_{n}>q_{N}^{\sigma_{n}}$. Il suffit de prendre $i=N$, car alors

$$
p_{n}=1+q_{1}^{\sigma_{n}}+\cdots+q_{N}^{\sigma_{n}}+\cdots+q_{n}^{\sigma_{n}}>q_{N}^{\sigma_{n}} .
$$

Si donc la condition $2^{\circ}$ était vérifiée, la suite $\sigma$ convergerait vers zéro.

Or on peut facilement trouver un point $x_{0}$ où $f_{p_{n}}^{n}(x)$ ne tend pas. vers zéro. En effet, on a évidemment

$$
f_{p}^{n}(x) \geqq 1 \quad \text { pour } \quad 0 \leqq \frac{r}{2^{n}}+\frac{1}{2^{n+p+2}} \leqq x \leqq \frac{r}{2^{n}}+\frac{3}{2^{n+p+2}} \leqq 1,
$$

quand $r$ est un entier quelconque prenant l'une des valeurs $0,1,2, \cdots, 2^{n}-1$. Appelons $I_{n, p}^{r}$ l'intervalle précédent. Il suffit alors de prouver qu'il existe un point $x_{0}$ commun à une infinité des intervalles $I_{1, p_{1}}^{r_{1}}, I_{2, p_{2}}^{r_{2}}, \cdots, I_{n, p_{n}}^{r_{n}}, \cdots$. Or étant donné l'intervalle $I_{n, p_{n}}^{r_{n}}$, prenons $n^{\prime}=n+p_{n}+2$, puis $r^{\prime}$ tel que

$$
\frac{r_{n}}{2^{n}}+\frac{1}{2^{n+p_{n}+2}}=\frac{r^{\prime}}{2^{n^{\prime}}}<\frac{r^{\prime}+1}{2^{n^{\prime}}}<\frac{r_{n}}{2^{n}}+\frac{3}{2^{n+p_{n}+2}} .
$$

Alors $I_{n^{\prime}, p_{n^{\prime}}^{\prime}}^{r^{\prime}}$ est compris dans $I_{n, p_{n}}^{r_{n}}$. On a donc une succession d'intervalles pris dans la suite des $I_{n, p_{n}}^{r_{n}}$ et chacun compris dans le précédent. Ils auront donc un point commun $x_{0}$.

12. La réponse à laseconde question $d u n^{\circ} 9$ est aussi négative. Nous allons en effet donner l'exemple d'une classe $(V)$ d'éléments où la limite est définie au moyen du voisinage et ne peut être définie sans altération au moyen d'un écart.

Considérons en effet la classe $(V)$ formée des points d'un segment $a b$ et où le voisinage d'un point d'abscisse $x_{0}$ est défini de la façon suivante. On appellera $T_{0}^{x_{0}}$ l'ensemble des points du segment $a b$ et $T_{n}^{x_{0}}$ (pour $n$ entier $\geqq 1$ ) l'ensemble des points du segment de longueur $1 / n$ ayant $x_{0}$ pour extrémité droite. Chacun de ces ensembles $T_{n}^{x_{0}}$ contient $x_{0}$ et contient le suivant $T_{n+1}^{x_{0}}$.

On voit évidemment que dans cette classe pour qu'une suite $\left\{x_{p}\right\}$ converge vers $x_{0}$, il faut et il suffit que la distance géométrique $x_{0} x_{p}$ tende vers zéro et de plus que les points $x_{p}$ soient à partir d'un certain rang à gauche de $x_{0}$ (ou confondus avec $x_{0}$ ).

Il s'agit de montrer qu'aucune définition d'un écart $\left(x, x^{\prime}\right)$ dans cette classe ne peut conduire à la même définition de la convergence.

En effet supposons une telle définition possible. Alors si $x^{\prime}$ est un point arbitraire à droite d'un point fixe $x$ l'écart $\left(x, x^{\prime}\right)$ aura une borne infoérieure positive. En effet, dans le cas contraire, cette borne serait nulle et on pourrait, quel que soit $n$, trouver un point $x_{n}$ à droite de $x$ tel que $\left(x, x_{n}\right)<1 / n$. Par suite la suite $\left\{x_{n}\right\}$ située à droite de $x$, devrait converger vers $x$ dans la 
classe, ce qui est contraire à la définition actuelle de la limite. Soit donc $F(x)$ la borne inférieure positive considérée, bien déterminée pour chaque valeur de $x$ dans $a b$.

Pour tout point $x_{0}$ de $a b$ et toute valeur de l'entier $p$, on peut déterminer un intervalle $x_{0}^{\prime} x_{0}$ limité à droite à ce point $x_{0}$ et à l'intérieur duquel $F(x)$ $<1 / p$. Car il suffit à cet effet de déterminer le point $x_{0}^{\prime}$ de sorte que pour $x_{0}^{\prime}<x<x_{0}$, on ait $\left(x, x_{0}\right)<1 / p$. Prenons en particulier $x_{0}=b$; on a un intervalle $x_{0}^{\prime \prime} b$ dans lequel $F(x)<1 / p$. Soit $\alpha$ la borne inférieure des nombres $x_{0}^{\prime \prime \prime}$ telle que dans l'intervalle $x_{0}^{\prime \prime \prime} b$, on ait $F(x)<1 / p$, sauf peut être en un ensemble dénombrable de points (il y a au moins un de ces intervalles, à savoir $\left.x_{0}^{\prime \prime} b\right)$. On a nécessairement $\alpha=a$. Car si $a<\alpha$, il suffirait de former à gauche de $\alpha$ comme plus haut un intervalle $\beta \alpha$ dans tout l'intérieur duquel $F(x)<1 / p$. Et alors l'intervalle $\beta b=\beta \alpha+\alpha b$ serait un intervalle plus grand que $\alpha b$, où $F(x)<1 / p$ sauf en un ensemble dénombrable de points.

Mais alors on voit que $F(x)$ ne peut être $\geqq 1 / p$ qu'en un ensemble dénombrable $E_{p}$ de points de $a b$. Par suite $F(x)$ ne pourrait être positif que dans l'ensemble dénombrable de points qui est la somme des ensembles dénombrables $E_{1}+E_{2}+\cdots+E_{p}+\cdots$, et non en tout point de $a b$.

13. On peut remarquer que dans le cas d'une classe $(V)$, on pourrait déterminer une sorte d'écart dissymétrique. Appelons en effet $((A, B))$ l'inverse de la plus haute valeur de $n$ telle que le voisinage $T_{n}^{A}$ contienne $B$. Ce nombre est $\geqq 0$, fini et bien déterminé si $A$ et $B$ sont distincts. Nous poserons $((A, B))=0$ si $A$ et $B$ coïncident. On voit qu'en général on aura $((A, B)) \neq((B, A))$. Cependant, $A$ étant un élément fixe, si $B_{p}$ tend vers $A,\left(\left(A, B_{p}\right)\right),-$ mais non nécessairement $\left(\left(B_{p}, A\right)\right)$,-tend vers zéro, et réciproquement.

14. Étant prouvé qu'une classe $(V)$ n'est pas nécessairement une classe $(E)$, nous allons déterminer maintenant à quelle condition il en est ainsi.

Nous supposons donc que dans une classe $(V)$, la limite soit définie en attachant à chaque élément $A$ une suite d'ensembles $T_{1}^{(A)}, T_{2}^{(\mathcal{A})}, \ldots, T_{n}^{(\mathcal{A})}$, $\ldots$, définissant des voisinages de plus en plus étroits. Et nous cherchons à quelle condition doivent satisfaire ces ensembles $T_{n}^{(A)}$ pour que la limite ainsi définie puisse être définie sans altération de la convergence ni de la limite par le moyen d'un écart $(A, B)$ attribué à chaque couple d'éléments $A, B$, $C$, cet écart satisfaisant aux conditions $1^{\circ}, 2^{\circ}$ du $\mathrm{n}^{\circ} 10$.

Cette condition est la suivante: il faut et il suffit que l'on puisse attacher à chaque élément $A$ une suite d'entiers non décroissants et qui tendent vers l'infini

$$
1=r_{1}^{A} \leqq r_{2}^{A} \leqq \cdots \leqq r_{n}^{A} \leqq \cdots,
$$

choisis de sorte que si l'on se donne arbitrairement un entier $N$ et un élé- 
ment $A$ de la classe, on puisse déterminer un entier $m$ pour lequel l'ensemble $T_{r_{N}^{B}}^{(B)}$ contient nécessairement l'élément $A$, si $B$ appartient à $T_{m}^{(\boldsymbol{A})}$.

La condition est nécessaire. En effet, soit une classe $(V)$ où la limite peut aussi être déterminée par le moyen d'un ecart $(A, B)$.

Comme on peut supposer que $T_{1}^{(\boldsymbol{A})}$ contient toute la classe, alors, pour chaque valeur de $n$, l'un au moins des ensembles $T_{1}^{(A)}, T_{2}^{(A)}, \cdots$, contient tous les éléments $B$ tel que $(A, B)<1 / n$. Si $T_{n}^{(A)}$ est précisément l'un de ces ensembles nous poserons $r_{n}^{A}=n$; sinon nous appellerons $r_{n}^{A}$ le rang le plus haut $(<n)$ de tous ces ensembles.

On voit que dans tous les cas $T_{r_{n}^{A}}^{(A)}$ contient tous les éléments $B$ tels que $(B, A)<1 / n$ et que $r_{n}^{A} \leqq r_{n+1}^{A}$. On voit aussi que $r_{n}^{A}$ tend vers l'infini quand $A$ est fixe; sans quoi il aurait une borne supérieure finie $k$. Et alors quel que soit $n>k$ il y aurait en dehors de $T_{k+1}^{(A)}$ un élément $B_{n}$ tel que $\left(B_{n}, A\right)$ $<1 / n$. Mais alors si la limite est définie par l'écart, $B_{n}$ tendrait vers $A$, et si elle est définie par le voisinage, $B_{n}$ ne tendrait pas vers $A$.

Ceci étant donnons nous arbitrairement $N$ et $A$. On peut choisir un entier $m$ tel que pour tout élément $B$ de $T_{m}^{(A)}$, on ait $(B, A)<1 / N$. (Car dans le cas contraire, il y aurait dans chaque ensemble $T_{m}^{(A)}$ un élément $C_{m}$ tel que $\left(C_{m}, A\right)>1 / N$. Alors selon qu'on emploierait le voisinage ou l'écart $C_{m}$ tendrait ou ne tendrait pas vers $A$.)

Or si $(B, A)<1 / N, T_{r_{N}^{B}}^{(\boldsymbol{B})}$ contient $A$. La condition est bien nécessaire.

Inversement, supposons que dans une classe $(V)$ l'existence des suites $r_{n}^{\boldsymbol{A}}$ satisfaisant aux conditions demandées soit assurée. Montrons qu'on peut aussi définir la limite dans la classe, sans altérer la convergence, au moyen d'un écart.

Pour cela appelons $q_{B}^{A}$ la plus grande valeur de $n$ telle que $T_{r_{n}^{A}}^{(A)}$ contient $B$. Il y a au moins une telle valeur de $n$ puisque $T_{r_{1}^{A}}^{(A)}=T_{1}^{(A)}$ est formé de toute la classe. Alors $q_{B}^{A}$ est un nombre bien déterminé et fini si $A$ et $B$ sont distincts. Posons alors $(A, B)=(B, A)=0$ si $A$ et $B$ coïncident et

$$
(A, B)=(B, A)=\frac{1}{q_{A}^{B}}+\frac{1}{q_{B}^{A}}
$$

dans le cas où ils sont distincts. Alors on a défini pour tout couple d'éléments un nombre $\geqq 0$ qui n'est nul que si les deux éléments coïncident. Il s'agit de montrer que si une suite $B_{p}$ tend vers $A,\left(B_{p}, A\right)$ tend vers zéro et réciproquement.

Tout d'abord si $\left(B_{p}, A\right)$ tend vers zéro, $q_{B_{p}}^{A}$ croît indéfiniment; donc, si $N$ est donné, on a pour $p$ assez grand $(p>m): q_{B_{p}}^{A}>N$; donc $T_{r_{N}^{A}}^{(A)}$ contient $B_{p}$, par définition de $q_{\boldsymbol{B}_{p}}^{\boldsymbol{A}}$. Mais si on s'est donné $A$ et un entier $\alpha$, on peut choisir $N$ pour que $r_{N}^{A}>\alpha$ et par suite $T_{a}^{(A)}$ contient aussi $B_{p}$ à partir du rang $m$. Autrement dit, puisque $\alpha$ est arbitraire, $B_{p}$ tend vers $A$. 
Inversement si $B_{p}$ tend vers $A$, et si $\alpha$ est un entier donné, $B_{p}$ est dans $T_{r_{a}^{A}}^{(A)}$ à partir d'un certain rang $k$; donc $q_{B_{p}}^{A}>\alpha$ pour $p>k$. Donc $q_{B_{p}}^{A}$ tend vers l'infini avec $p$. Pour montrer que $\left(A, B_{p}\right)$ tend vers zéro, il suffit donc de montrer que $q_{A}^{B_{p}}$ tend aussi vers l'infini. Or dans le cas contraire on pourrait trouver un nombre positif $\lambda$ tel que pour une infinité de valeurs de $p$, on ait $q_{A}^{B_{p}}<\lambda$. Mais pour une telle valeur de $p, T_{r_{\lambda}^{B p}}^{\left(B_{p}\right)}$ ne contiendrait pas $A$. Or par hypothèse, étant donnés $A$ et $\lambda$ on peut trouver un entier $m$ tel que si $B_{p}$ appartient à $T_{m}^{(A)}, T_{r_{\lambda}^{B}}^{\left(B_{p}\right)}$ contienne $A$. Il faudrait donc que pour une infinité de valeurs de $p, B_{p}$ n'appartienne pas à $T_{m}^{(A)}$, ce qui est contraire à la définition de la limite par le voisinage.

15. Pour élucider complètement les relations entre les notions de limite et de distance, il reste à résoudre le problème suivant:

Nous avons vu que pour qu'une classe $(L)$ soit une classe $(D)$, il faut qu'elle soit $(S)$ et $(E)$. Ces deux conditions nécessaires, qui sont indépendantes, sont elles suffisantes? Et dans le cas de la négative, quelle est la nature de la condition supplémentaire?

Ce problème n'est pas résolu dans le présent mémoire. Nous nous contenterons des indications suivantes.

16. On peut sérier les difficultés en passant du cas des classes qui sont à la fois $(E)$ et $(S)$ au cas des classes $(D)$ par l'intermédiaire des classes que j'ai appelées $(V)$ dans ma Thèse, notation à laquelle je n'attache plus ici le même sens, le terme voisinage se trouvant plus adéquatement utilisé dans le présent mémoire. Nous appellerons ces classes non plus $(V)$, mais $\left(E_{r}\right)$, et nous dirons que l'écart dans ces classes est uniformément régulier. Les considérations suivantes vont expliquer la raison de ces dénominations et pourquoi les classes $\left(E_{r}\right)$ s'introduisent naturellement dans la solution $\mathrm{du}$ problème que nous venons de poser.

17. Voyons donc quelles seraient les propriétés d'une classe qui seràit a la fois $(E)$ et $(S)$. Les conditions $1^{\circ}$ et $2^{\circ}$ vérifiées par une classe $D$ restant remplies par une telle classe, il s'agit de savoir ce qui remplace la condition $3^{\circ}$. A cet effet démontrons le théorème suivant:

La condition nécessaire et suffisante pour qu'une classe $(E)$ soit $(S)$, (autrement dit pour que tout ensemble dérivé soit fermé dans une classe où la limite est définie à l'aide de l'écart) est qu'à tout élément $A$ et à tout nombre positif $\delta$, on puisse faire correspondre un nombre $\eta$ tel que si $B$ est un élément déterminé dont l'écart avec $A$ soit moindre que $\eta$ et si $\omega$ est un nombre positif convenablement choisi une fois connus $\delta, \eta, A, B$, alors pour tout élément $C$ dont l'écart avec $B$ est moindre que $\omega$, on aura*

$$
(A, C)<\delta \text {. }
$$

* On peut exprimer brièvement cette condition en disant que si $(A, B)$ et $(B, C)$ sont des infiniments petits, $(A, C)$ est aussi un infiniment petit. Mais la convergence n'est pas nécéssairement uniforme. 
En effet supposons d'abord que la classe soit $(E)$ et $(S)$. Si le théorème n'était pas vrai pour un élément $A$ de l'ensemble et un certain nombre $\delta>0$, la condition $(A, C)<\delta$ ne serait pas nécessairement vérifiée et ceci quelle que soit la valeur adoptée pour $\eta$. En particulier si l'on prenait $\eta=1 / n$, il y aurait au moins un élément $B_{n}$ tel que $\left(A, B_{n}\right)<1 / n$, pour lequel aucun choix convenable de $\omega$ ne saurait assurer la condition recherchée. C'est à dire que si l'on prenait par exemple $\omega=1 / p$, il y aurait un élément $C_{p}^{(n)}$ tel que

$$
\left(B_{n}, C_{p}^{(n)}\right)<\omega=\frac{1}{p} \quad \text { et } \quad\left(A, C_{p}^{(n)}\right)>\delta .
$$

Mais, quand $n$ restant fixe $p$ croît indéfiniment, $C_{p}^{(n)}$ tend vers $B_{n}$. Et quand $n$ croît indéfiniment, $B_{n}$ tend vers $A$. L'ensemble dérivé de l'ensemble des $C_{p}^{(n)}$ comprend donc l'ensemble des $B_{n}$. Et comme il est fermé par hypothèse, il comprend aussi la limite $A$ des $B_{n}$. Or la condition $\left(A, C_{p}^{(n)}\right)>\delta>0$ montre que $A$ ne peut être limite d'une suite d'éléments $C_{p}^{(n)}$.

Réciproquement supposons que pour une classe $(E)$ la condition énoncée plus haut soit vérifiée. Alors tout ensemble $E^{\prime}$ dérivé d'un ensemble quelconque d'éléments de la classe est fermé. Autrement dit, si $A$ est la limite d'une suite d'éléments $B_{1}, B_{2}, \cdots, B_{n}, \cdots$, de l'ensemble dérivé $E^{\prime}, A$ appartient à $E^{\prime}$. En effet, chaque élément $B_{n}$ de $E^{\prime}$ est la limite d'une suite d'éléments $C_{1}^{(n)}, C_{2}^{(n)}, \ldots$. Pour $\delta$ donné, on peut trouver un nombre $\eta$ tel que si $\left(A, B_{n}\right)<\eta$, il correspond à $B_{n}$ un nombre $\omega_{n}$ pour lequel la condition $\left(B_{n}, C_{p}^{(n)}\right)<\omega_{n}$ entraine $\left(A, C_{p}^{(n)}\right)<\delta$. Or quel que soit $\eta$ on peut prendre $n$ assez grand pour que $\left(A, B_{n}\right)<\eta$, et quel que soit $\omega_{n}$, on peut prendre pour $n$ fixe $p$ assez grand pour que $\left(B_{n}, C_{p}^{(n)}\right)<\omega_{n}$. Les $C_{1}^{(n)}, C_{2}^{(n)}, \ldots$ étant distincts ainsi que les $B_{1}, B_{2}, \cdots$, on voit qu'on peut finalement faire correspondre à toute valeur $1 / i$ de $\delta$ deux entiers $n_{i}, p_{i}$, tels que les éléments $C_{p_{1}}^{\left(n_{1}\right)}, C_{p_{2}}^{\left(n_{2}\right)}, \cdots$ soient distincts et que $\left(A, C_{p_{i}}^{\left(n_{1}\right)}\right)<1 / i$. Autrement dit, $A$ appartient aussi à $E^{\prime}$.

18. Si le nombre $\omega$ ne dépend pas de l'élément $B$, alors en prenant pour $\epsilon$ le plus petit des nombres $\eta$ et $\omega$, on voit qu'à tout élément $A$ et à tout nombre $\delta>0$, on peut faire correspondre un nombre $\epsilon$ tel que si $B, C$ sont deux éléments satisfaisant à la condition

$$
(A, B)+(B, C)<\epsilon \text {, et par suite à }(A, B)<\eta,(B, C)<\omega,
$$

on a toujours

$$
(A, C)<\delta .
$$

Appelons $\psi(\theta, A)$ la borne supérieure, finie ou non, de $(A, C)$ quand $B, C$ sont des éléments quelconques tels que $(A, B)+(B, C)=\theta>0$. On voit alors que $\psi(\theta, A)$ est un nombre $>0$ qui tend vers zéro quand $\theta$ tend 
vers zéro, $A$ restant fixe. Car quel que soit le nombre $\delta>0$ on peut trouver un nombre $\theta$ tel que si $(A, B)+(B, C)<\epsilon$, on a $(A, C)<\delta$, et par suite si $\theta<\epsilon, \psi(\theta, A) \leqq \delta$. Et on a quels que soient $B$ et $C$

$$
(A, C) \leqq \psi((A, B)+(B, C), A) \text {. }
$$

Si maintenant on suppose que $\psi(\theta, A)$ est aussi indépendant de $A$, on obtient une classe qui est ce que nous appellerons une classe $\left(E_{r}\right)$, où on peut définir un écart uniformément régulier tel que l'on ait, quels que soient $A, B, C$,

$$
(A, C) \leqq \psi((A, B)+(B, C)) .
$$

On voit que les classes $(D)$, celles où la distance est définie, correspondent au cas particulier des classes $\left(E_{r}\right)$ où $\psi(\theta)=\theta$. Il faut remarquer d'ailleurs qu'on a toujours $\psi(\theta) \geqq \theta$ comme on le voit en prenant $C$ identique à $B$.

Ces classes $\left(E_{r}\right)$ ne sont autres que celles que j'ai appelées classes $(V)$ dans ma Thèse. On voit en effet que si l'on appelle $\phi(\epsilon)$ une fonction partout supérieure à $\psi(2 \epsilon)$ mais tendant vers zéro, on aura

$$
(A, C)<\phi(\epsilon) \text { pour }(A, B)<\epsilon,(B, C)<\epsilon .
$$

\section{Notes SUpplementaires.*}

$A$. Pour obtenir des distances infiniment petites, il suffirait de faire correspondre la classe à un ensemble fini linéaire non isolé avec conservation de la distance par définition. Et au cas où la puissance de la classe est supérieure au continu, de donner aux distances aboutissant aux éléments restants une valeur finie suffisamment grande.

$B$. Dans une note récente aux Comptes-Rendus de l'Académie des Sciences (Septembre, 1917) je généralise une nouvelle fois en abandonnant la condition que les ensembles $U$ forment une suite dénombrable.

$C$. Il en résultera, en particulier, que dans cette classe si importante on ne peut definir un écart. On peut, d'ailleurs, donner de ce fait démonstrations directes plus simples. J'ai aussi fait remarquer, dans ma Thèse, que cette classe n'est pas une classe $(s)$.

* These notes were received from the author too late to insert with the pages to which they belong.-THE Editors. 